
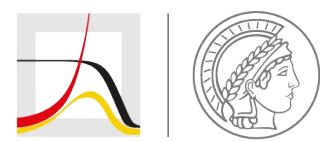

MAX PLANCK INSTITUTE

FOR DEMOGRAPHIC RESEARCH

Konrad-Zuse-Strasse 1 · D-18057 Rostock · Germany · Tel +49 (0) 3812081 - 0 · Fax +49 (0) 3812081 - $202 \cdot$ www.demogr.mpg.de

MPIDR Working Paper WP 2020-022 I May 2020

https://doi.org/10.4054/MPIDR-WP-2020-022

\title{
The Impact of Demographic Change on Transfers of Care and Associated Well-being
}

\author{
Denys Dukhovnov \\ Joan M. Ryan \\ Emilio Zagheni I sekzagheni@demogr.mpg.de
}

(c) Copyright is held by the authors.

Working papers of the Max Planck Institute for Demographic Research receive only limited review. Views or opinions expressed in working papers are attributable to the authors and do not necessarily reflect those of the Institute. 
Denys Dukhovnov, University of California, Berkeley, Berkeley, CA, USA

Joan M. Ryan, University of Pennsylvania, Philadelphia, PA, USA

Emilio Zagheni, Max Plank Institute for Demographic Research, Rostock, Germany

\title{
WORKING PAPER
}

\section{The Impact of Demographic Change on Transfers of Care and Associated Well-being}

\begin{abstract}
Objectives: This study aims to evaluate the impact of demographic change on long-term, macrolevel childcare and adult care transfers, and the associated well-being effects of informal caregiving.

Method: We measure the impact of demographic change on non-monetary care exchanged between different groups by estimating matrices of time transfers by age and sex, and weighting the time flows by self-reported indicators of well-being, for activities related to childcare and adult care. The analysis employs cross-sectional data from the American Time Use Survey 2011-2013, and the Panel Study of Income Dynamics, Disability and Use of Time Module 2013 to produce the estimates of well-being associated with various forms of care.

Results: We show that people have more positive feelings when caring for children than when caring for adults. Although reductions in the country-level care supply are expected to be small relative to demand, future projections indicate a $17.1 \%$ decrease in the ratio of time spent caring for children under age 15 relative to time spent caring for the rest of the population by 2050 . While this change is expected to produce only a minor increase in the ratio of negative-to-positive feelings associated with caregiving, purely due to population aging, it could have nontrivial deterioration of well-being for some caregivers.
\end{abstract}

Discussion: Significant reductions in absolute caregiver well-being caused by demographic changes at the population level may reduce workload, productivity, and adversely impact health, if not offset by caregiver-friendly family policies.

Key Words: household production, caregiving, time use 


\section{Introduction}

Intergenerational transfers in the form of informal care - which can be seen as a major intangible resource - are strongly affected by demographic changes such as lower and later fertility, as well as reduced mortality and population aging. Changes in the population age structure determine the fraction of the population in each stage of the life course. Thus, the composition of the population has macro-social and economic consequences for the flow of resources to childcare and adult care arrangements. A limitation of the approaches that have so far been used to study such non-monetary transfers is that they do not distinguish between the levels of well-being associated with performing different activities that involve transfers of unpaid resources, such as household chores or eldercare. As there is no clear way to assign a dollar value to the opportunity costs of such interchangeable unpaid activities, a minute spent on one such activity is often assumed to be indistinguishable from a minute spent on another. While this assumption may be reasonable in a number of situations, in other circumstances the levels of well-being associated with performing specific informal care activities may vary substantially. Population aging is a phenomenon that creates challenges for most developed societies, the implications of which extend beyond ensuring that there is adequate public funding in the form of medical care, financial support, paid services, or other material transfers for children and the elderly (Kohli, 1999). Informal family support, usually in the form of unpaid care, can be seen as an invisible backbone that affects the lives of a vast number of families with dependent and ailing members. However, the amounts of informal support provided by family members are poorly documented by conventional resource-flow measurement mechanisms (Gál et al., 2015). The demand for nonmonetary support, which is characterized by transfers of non-tangible resources between various

population groups, is increasing. However, because of the complexity and the physical and 
psychological pressures involved in providing informal care, individuals with family members who need care often partially substitute or outsource their care responsibilities, particularly if their relatives require long-term eldercare or have high levels of disability (Van Houtven \& Norton, 2004; Bonsang, 2009).

At the same time, a family's financial assets are not a strictly limiting factor in many care arrangements. Most studies on the determinants of the prevalence of household production have focused on the socio-cultural factors underlying the gender divide, family resources, or labor market conditions (Shire et al., 2017). Indeed, the well-being of the caregiver is a crucial component in the process of deciding who will provide care, and in regulating the utilization of paid care services and informal care work (Kossek et al., 2001). While physically demanding, providing childcare is generally seen as fulfilling and meaningful in the long run because the caregivers are able to see their efforts come to fruition when the children succeed later in life (Hammersmith \& Lin, 2015). By contrast, providing adult care, particularly in the form of eldercare, is usually regarded as much more challenging for caregivers. It has, for example, been shown that providing informal eldercare can lead to substantial work strain for caregivers, in part due to competing demands (Trukeschitz et al., 2012); and that it involves greater physical burdens than caring for children (de Oliveira et al., 2015). Moreover, it appears that the stresses experienced by caregivers can differ depending on their age and sex. For instance, women, as mothers, are usually the primary caregivers for their own young children (Nomaguchi et al., 2005); but as longevity increases, women are also spending more time caring for elderly parents, grandparents, or spouses (Wiemers \& Bianchi, 2015).

The demographic structure of the US population is likely to change in the future, given that currently in the US, fertility has fallen below the replacement level (Martin et al., 2018), and life 
expectancy at birth has risen to 78 years (Kochanek et al., 2019). It may be expected that these demographic shifts will increase the dependency of the population on eldercare services financed by private and public monetary transfers that are already facing challenges (Lee \& Mason, 2017). However, in order to accurately project the exact dependency balance, modeling is needed that accounts for a multitude of differentiating factors, such as socioeconomic background, social support networks, and the well-being of caregivers engaged in informal intergenerational transfers. For many countries, the formal side of this support mechanism has been investigated relatively well by the National Transfer Accounts (NTA) project, and by the UN System of National Accounts (SNA) (Lee \& Mason, 2011). Our present contribution consists of documenting and forecasting the amount and the character of the informal care time transferred in the United States, with weights that introduce a qualitative component reflecting the caregivers' physical and emotional well-being. We formulate the two interdependent research questions:

1. Given that, in general, the condition of the elderly progressively deteriorates relative to growing children, is providing eldercare significantly associated with a change across multiple stressors of caregiver well-being? If so, how positive or negative is the net impact for an average caregiver in the population?

2. The well-being of informal caregivers can mediate the decisions of individuals to provide care and thus the cumulative share of unpaid care in the national care supply, tipping the balance of formal and informal care markets for the dependent groups. In this light, as the population progressively ages, what effect would the future changes in the population structure bring about in terms of the aggregate unpaid caregiver well-being? What may this entail for the future informal care supply. 
To answer these questions, we first describe our data and the approach we use to estimate matrices of time transfers by age and sex, while incorporating caregiver well-being. We then present our results and demonstrate the projected shift in informal care dependency, driven by compositional changes in the population. In the final section, we conclude with a discussion of our results that includes suggestions for future research in the area of intergenerational economy.

\section{Design and Methods}

Data

Our analysis was based on data from the American Time Use Survey (ATUS) and the Panel Study of Income Dynamics (PSID). Specifically, we used data from the ATUS (2011-2013) to produce matrices of transfers of care time, by age and sex. The ATUS, which is the most authoritative study of how people spend their time in the US, features a representative sample of about 12,000 participants selected annually from the Current Population Survey (CPS) respondents. The data provide a detailed, chronological account of the respondents' activities over a randomly selected day, including information about the other people who were present at the activities, and the duration and the location of the activities. In addition to data from the main questionnaire, we used data from the Well-Being Module for years 2012-2013. The module measures the emotional and physical impact of participation in a range of activities, the duration and the circumstances of which were assessed in the main questionnaire. Unlike the main ATUS questionnaire, the WellBeing Module consists of sets of exactly three randomly selected activities from the pool of eligible activities per respondent. The six feeling indicators measured across all activities and available in the Well-Being Module are happiness, meaning, pain, sadness, stress, and tiredness. Each of these indicators is on a seven-point Likert scale that ranges from zero to six, with zero representing the 
lowest intensity, and six representing the highest intensity. We employed the combined samples of the 2012 and 2013 Well-Being Module questionnaires to ensure that our sample size was adequate. However, ATUS carries an important limitation. The identification and classification of inter-household transfers of care time within activities by age and sex of care recipients is limited only to own non-household children and some elderly who receive informal care due to a health condition related to aging.

In order to address this limitation and to obtain more robust estimates for the entire crosssection of the population, we complemented the ATUS data with data extracted from the PSID Disability and Use of Time supplement (DUST). PSID included in its 2013 wave detailed 24-hour time diaries of respondents aged 60 and older, along with those of their spouse or partner. The inclusion of PSID data into our analysis thus ensured that we had adequate sample size to draw conclusions about the non-household transfers to and by older adults. Moreover, when combined with detailed disability measures linked to the main PSID questionnaire, the DUST data can provide greater insights into the factors that promote subjective well-being among older adults experiencing functional losses, and among the individuals providing them with assistance. The pool of respondents we studied was limited to those who indicated that they were engaging in caregiving. We divided our sample into those who were caring for children and those who were caring for adults based on their answers to two binary questions: one that asked whether they were caring for a child, and a second that asked whether they were caring for an adult. We examined the respondents' self-reported levels of happiness, calmness, frustration, worry, sadness, tiredness, and pain associated with providing childcare or adult care, all recorded on a 0-6 Likert scale.

Matrices of Time Transfers by Age and Sex 
Matrices of intra-household flows of care time could be estimated directly from the time use diaries, as the respondents recorded the time they dedicated to various caregiving activities, as well as the unique identifiers of the household members who benefited from this time. Inter-household transfers could not be estimated directly because records of the age and sex of the care recipients were lacking. Inter-household flows were instead obtained indirectly by combining the available information about the time the respondents dedicated to inter-household care activities, as reported in their diaries, with the frequencies of care recipients in various age and sex groups assigned to the caregivers in the ATUS Eldercare Roster. The matrices of intra- and inter-household time transfers were subsequently combined into a single tabulation of overall care time transfers. The derivation and estimation of the matrices are not central for the present analysis. For details about the matrix estimation methods, see Section 1 of the supplementary materials in our previous work (Blinded for Review).

We employ age-sex entries of these matrices as multipliers to calculate the projected amount of positive and negative time spent providing care on the national level to corroborate our second research question. However, we first needed to calculate the indices of positivity and negativity with regard to the caregiver well-being.

\section{Indices of Positivity and Negativity Associated with Childcare and Adult Care}

We combined the information on feelings in the ATUS and PSID data to calculate indices of positivity and negativity associated with activities related to childcare and adult care. We then weighted the transfer matrices using these indices. First, we grouped the well-being states in the ATUS and PSID data into positive and negative classes. For each subset of the data, we calculated mean scores for every well-being state. For positive feelings (ATUS: happiness and meaningfulness; PSID: happiness and calmness), six was the most positive value. For negative 
feelings (ATUS: pain, sadness, stress, tiredness; PSID: frustration, worry, sadness, tiredness, pain), six was the extreme negative value. For negative states, the calculated difference between six and the reported score indicated a "lack of negativity." By applying this procedure to all of the wellbeing states, whether negative or positive, we ended up with a scale from zero to six, with zero indicating high negativity and six indicating high positivity. Next, we computed the average positivity across all of the well-being states in each dataset (six in ATUS andseven in PSID), and weighted those values by the sample size of each dataset (ATUS: 3,225 for childcare, 526 for adult care; PSID: 264 for childcare, 243 for adult care) in order to reflect differences in uncertainty with respect to the estimates from the two datasets. Finally, we rescaled the obtained averages to obtain negativity and positivity ratios, such that childcare and adult care negativity and positivity summed up to one for each care activity. The overall indices for emotional states related to childcare and adult care based on the combined ATUS and PSID data are reported in Table 1.

\section{[TABLE 1]}

\section{Results}

Several important features stand out in our estimates of overall flows of time spent caregiving by age and sex. First, as Figure 1 shows, the majority of time was transferred from parents to young children: i.e., on average, for every minute spent providing care to a person aged 65 or older, around three minutes were spent providing care for children under age 15 . These findings differ markedly by sex, as women spent about twice as much time caring for young children as men. Second, gender differences in transfers from grandparents to grandchildren are quite noticeable, as the grandfathers reported spending more time with their grandsons and the grandmothers reported spending more time with their granddaughters. 


\section{[FIGURE 1]}

Third, we observed a ridge along the main diagonal of the matrix of transfers for people of the opposite sex, indicating substantial transfers to spouses. In addition, we found evidence of sex differences in the time dedicated to caring for the elderly and care time needed by the elderly. For instance, elderly women appeared to have slightly greater care needs than elderly men, which may be explained by women's higher relative life expectancy. The analysis also showed that middleaged women spent slightly more time caring for the elderly than middle-aged men.

\section{Well-being Associated with Childcare and Adult Care}

In terms of well-being, informal caregivers engaging in childcare reported very high levels of happiness and meaning, with average scores of 5.0 and 5.42, respectively. The scores associated with adult care were lower, trailing the scores associated with childcare by an average of 0.66 scale points for happiness and 0.58 scale points for meaning. Conversely, respondents reported higher intensities of negative feelings such as pain, sadness, and stress when performing adult care duties than when engaging in childcare. Although these measures received high average ratings - below 1.0 for pain and sadness, and 2.5 or less for stress and tiredness - levels of negative emotions and dissatisfaction were higher for adult care than for childcare activities. For tiredness, respondents engaged in childcare rated their tiredness slightly higher, with a mean score of 2.51 , whereas those engaged in adult care activities rated their tiredness somewhat lower, with a mean score of 2.11 . Compared to engaging in several other types of activities, such as household work, leisure, and main job activities, engaging in childcare was rated higher in terms of happiness and meaning, but also as more tiring.

The difference in the distribution of weighted means of childcare and adult care across various emotions and physical states was highly statistically significant $(|t|>4, p<0.001)$ for all 
but pain $(t=-1.46, p=0.145)$. Figure 2 shows the average scores of the feelings associated with informal care, and the outcome of the test of significance for the differences in the average feeling scores related to childcare and adult care. For example, engaging in childcare ranked significantly higher on the happiness and meaning scales (4.86 and 5.22, respectively) than engaging in adult care (4.41 and 4.66), leisure activities (4.47 and 4.05), household chores (4.22 and 4.24), and main job activities (3.99 and 4.20). Thus, performing adult care was rated significantly higher in terms of happiness and meaning than engaging in household chores or main job activities. Among the negative feelings, it is worth noting that for sadness, engaging in childcare was rated the lowest, at 0.36 ; while engaging in adult care was rated the highest, at 0.87 . The remaining comparison activities were not statistically significant from one another. For the remaining negative feelings, the scores associated with performing childcare and adult care were not significantly distinct from the scores associated with engaging in leisure, main job activities, and household chore activities. Data from the PSID confirmed that there were indeed significant differences in the feelings associated with engaging in childcare and adult care. The respondents reported, on average, more positive feelings (happy and calm) and fewer negative feelings (frustrated, worried, sad, tired, pain) when caring for children under age 18 than when caring for adults. Even with small sample sizes of 316 and 403, respectively, a difference-in-mean test indicated that all of the feelings except sadness were significantly different at the $95 \%$ confidence interval.

\section{[FIGURE 2]}

We also explored gender differences in feelings associated with care work. On the whole, women demonstrated higher mean values for happiness, calmness, sadness, tiredness, and pain when caring for children, and had higher mean values for frustration, worry, sadness, tiredness and pain when caring for adults. Selecting gender groups from within our sample pool meant that the 
sample sizes for further analyses were even more limited. A difference-in-mean test detected a statistically significant gender difference in pain only for respondents engaged in for adult care, as the only significant gender difference found for respondents engaged in childcare was for sadness.

To examine differences by age, we divided the respondents into nine age groups ranging from age 45 to age 85 or older. Among the respondents engaged in childcare, a general increase in happiness and calmness was found as the age of the caregiver increased. Frustration initially decreased with age, but spiked back up after caregivers turned age 75 . A similar trend was detected for tiredness, which may be because as caregivers grew older, they became physically less able to engage in high-energy childcare. The other negative feelings, such as worry, sadness, and pain, appeared to generally decrease as the age of the caregiver increased. Due to the small sample size in the PSID data, we were unable to provide a definitive statement about the association between the feelings and key demographic variables, like age and sex.

\section{Projections Using Age Profiles of Positive and Negative Time Transferred}

Profiles of positive and negative time production and consumption by age were obtained from the matrices weighted by positive and negative indices. These per capita profiles, shown in Figure 3, are the marginal row sums of each matrix. We encourage readers to consult Section 2 of supplementary materials for the detailed methodology on modeling transfer schedules by age (Author) and Section 3 for the details and descriptive results of transfers matrix decomposition into positive and negative flows. We proceed with implementing these intermediary components to produce future projections of care supply, adjusted for well-being.

Assuming the per capita production of each age group remains time-invariant, to evaluate the potential consequences of population aging on well-being related to caregiving activities, we 
combined our estimates of time transfers, weighted by the index of positivity and negativity, with age-specific population projections available in World Population Prospects for the years 20102100 (United Nations, 2019). As women and men engage in care differentially, we had to ensure that the aggregate care time was calculated separately for each gender before we added the values for the two genders together to obtain the aggregate care time for the entire population. We weighted the production side of care, while leaving the consumption side untouched, because the feelings used to generate the weights were reported only by the caregivers; that is, by the producers of care time.

\section{[FIGURE 3]}

Next, we defined the care support ratio (CSR) as a non-monetary alternative to the economic support ratio (SR) used by the National Transfer Accounts project community (Lee \& Mason, 2011). The positive CSR reflects the fraction of care time during which caregivers experienced positive emotions, divided by the total care time consumed, or received by care recipients. Similarly, the negative CSR indicates the fraction of care time during which caregivers experienced negative emotions, divided by the total time consumed. To calculate the positive and negative CSR, we kept the same denominator as in the traditional CSR; that is, the total amount of time received. The numerator was instead divided into two parts of positive time and negative time, which summed up to the total time spent by the caregivers. Figure 4 shows the estimated CSR, for which the indicators were designed so that the sum of the positive and negative CSR was equal to the total CSR. This outcome demonstrates what would happen to the ratio between the overall time produced by the caregivers and the time consumed by the care recipients if people continue to behave like they do today on a per capita basis, and the only change that occurs in the future is a demographic shift in which the population's age structure changes according to the 
medium-fertility variant UN projections. This hypothetical scenario indicates that the overall care dependency will decline, although not dramatically. However, population aging also implies that an increased proportion of transfers will be associated with negative feelings, as the positive CSR is expected to decline at a faster rate than the negative CSR. Although adjustments in the total time spent on the "production" of care are unlikely to be very large in the near future, the composition of the time transfers indicates that a deterioration in overall well-being associated with caregiving may be expected to occur solely due to demographic change.

\section{[FIGURE 4]}

\section{Discussion and Implications}

In this paper, we generated matrices indicating who transfers time to whom by age and sex, and weighted time flows by self-reported indicators of well-being for activities related to childcare and adult care. The empirical analysis, which was based on data from the American Time Use Survey and the Panel Study of Income Dynamics, confirmed our first hypothesis that people experience more positive feelings and fewer negative feelings when caring for children than when caring for adults. This pattern was reflected in the difference in per capita positivity associated with providing childcare and providing adult care. We also found evidence in favor of our second hypothesis, as the current care support ratio points to small reductions in this dynamic at the population level. A continuously aging population will mean that the proportion of transfers in the form of adult care will increase relative to the proportion of transfers in the form of childcare. In a similar vein, if engaging in adult care induces more negative feelings than engaging in childcare, we can expect that in the coming decades, the overall proportion of negative feelings will increase relative to the overall proportion of positive feelings. These findings suggest that in order to offset the future consequences of the increase in negativity due to caregiving, additional research on the factors that 
alleviate and mediate the stressors associated with care is needed. This trend may have significant health consequences at the population level, given that the degree of intensity and the duration of care can vary depending on the type of care provided, and that psychological stress appears to be more detrimental to the well-being of caregivers than physical tasks (Pinquart \& Sörensen, 2007). In particular, providing long-term intensive care for an elderly spouse with cognitive impairments has been shown to be very emotionally taxing (Kim et al., 2017). Though the increase in the prevalence of negative instances associated with informal care activities, such as tiredness and stress may not necessarily preclude caregivers from engaging in care thus reducing its output. However, the reduction of positive instances may present incentives to caregivers to avoid performing these activities themselves, or at all, for personal or purely demographic reasons (e.g. availability of kin). For instance, if the median age of a family were to increase, situations when working-age adults might choose not to devote additional time to playing with a child in lieu of caring for a disabled parent or relative might become more commonplace. In turn, on the national level the aggregate of such occasions will likely translate into partial substitution of informal care with formal care that will need to be planned and supplied through formal market mechanisms.

Our analysis sheds light on the interesting category of grandparenting, where older adults who are potential recipients of care are themselves engaging in caregiving. Several previous studies have investigated the emotional effects of grandparenting, and have demonstrated that a higher frequency of contact with grandchildren predicts higher levels of satisfaction among grandparents (Candida, 1999). We found this to be largely true, as caring for grandchildren was shown to be more satisfying than, for example, caring for an elderly spouse. However, custodial grandparenting can be harmful for psychosocial adjustment, and may increase the grandparents' need for mental health support, especially the grandchildren they are caring for are problematic 
(Emick \& Hayslip, 1996). Thus, the effect of providing childcare might differ between parents who are caring for their children and grandparents who are caring for their grandchildren. Demographic changes affecting trends in grandparenting necessitate another approach to studying these differences, separate from simply accounting for the number of grandparents. We further concur with statements made in the recent literature by emphasizing that mortality reductions will prolong grandparenthood, while fertility decline will decrease the proportion of people who ever become grandparents (Margolis, 2016).

In our analysis, we were only able to obtain a one-sided picture from the perspective of caregivers. Future work can also capitalize on the fact that the PSID data contain responses from both the head of the household and the head's spouse or partner. In the event that either party indicates having cared for the other, we can obtain feeling scores for both the caregiver and the care recipient that will enable us to gain further insight into the subjective feeling weights associated with the production and consumption of care time. While we also recognize that cultural considerations play a substantial role in caregivers' decisions and their feelings toward particular care activities, examining such interactions was beyond the scope of this paper.

\section{References}

Bonsang, E. (2009). Does informal care from children to their elderly parents substitute for formal care in Europe? Journal of health economics, 28(1), 143-154. doi:10.1016/j.jhealeco.2008.09.002 
Candida, C. P. (1999). Grandfathers and Grandmothers Satisfaction with the Grandparenting Role: Seeking New Answers to Old Questions. The International Journal of Aging and Human Development, 49(1), 61-78.

Emick, M. A., \& Hayslip, B. (1996). Custodial Grandparenting: New Roles for Middle-Aged and Older Adults. The International Journal of Aging and Human Development, 43(2), 135154. doi:10.2190/1fh2-ahwt-1q3j-pc1k

Gál, R. I., Szabó, E., \& Vargha, L. (2015). The age-profile of invisible transfers: The true size of asymmetry in inter-age reallocations. The Journal of the Economics of Ageing, 5, 98-104. doi:10.1016/j.jeoa.2014.09.010

Hammersmith, A. M., \& Lin I.-F. (2015, April 30 - May 2). Negative and Positive Experiences of Intergenerational Caregiving. Paper presented at the Annual Meeting of the Population Association of America 2015, San Diego, CA.

Kim, M. H., Dunkle, R. E., Lehning, A.J., Shen,H.-W., Feld, S., \& Perone, A. K. (2017). Caregiver stressors and depressive symptoms among older husbands and wives in the United States. Innovation in Aging, 1(suppl_1), 861-861.

doi:10.1093/geroni/igx004.3098

Kochanek, K. D., Murphy, S. L., Xu, J. Q., \& Arias, E. (2019) Deaths: Final data for 2017. National Vital Statistics Reports (vol. 68, no. 9). Hyattsville, MD: National Center for Health Statistics.

Kohli, M. (1999). Private and public transfers between generations: Linking the family and the state. European societies, 1(1), 81-104. doi:10.1080/14616696.1999.10749926 
Kossek, E. E., Colquitt, J. A., \& Noe, R. A. (2001). Caregiving decisions, well-being, and performance: The effects of place and provider as a function of dependent type and workfamily climates. Academy of management Journal, 44(1), 29-44. doi:10.5465/3069335

Lee, R. D., \& Mason, A. (Eds.). (2011). Population Aging and the Generational Economy: A Global Perspective. Edward Elgar Publishing. doi:10.4337/9780857930583

Lee, R. D., \& Mason, A. (2017). Cost of aging. Finance \& development, 54(1), 7.

Margolis, R. (2016). The Changing Demography of Grandparenthood. The Journal of Marriage and Family, 78(3), 610-622. doi:10.1111/jomf.12286

Martin, J. A., Hamilton, B. E., Osterman, M. J. K., Driscoll, A. K., \& Drake P. (2018) Births: Final data for 2017. National Vital Statistics Reports (vol. 67, no. 8). Hyattsville, MD: National Center for Health Statistics.

Mohide, E. A., Torrance, G. W., Streiner, D. L., Pringle, D. M., \& Gilbert, R. (1988). Measuring the Wellbeing of Family Caregivers Using the Time Trade-off Technique. Journal of Clinical Epidemiology, 41(5), 475-482. doi:10.1016/0895-4356(88)90049-2

Nomaguchi, K. M., Milkie, M.A., \& Bianchi, S. M. (2005). Time Strains and Psychological WellBeing: Do Dual-Earner Mothers and Fathers Differ? Journal of Family Issues, 26(6), 756792. doi:10.1177/0192513x05277524

de Oliveira, G. R., Neto, J. F., de Camargo, S. M., Lucchetti, A. L. G., Espinha, D. C. M., \& Lucchetti, G. (2015). Caregiving across the lifespan: comparing caregiver burden, mental health, and quality of life. Psychogeriatrics, 15(2), 123-132. doi:10.1111/psyg.12087

Pinquart, M., \& Sörensen, S. (2007). Correlates of physical health of informal caregivers: a metaanalysis. The Journals of Gerontology Series B: Psychological Sciences and Social Sciences, 62(2), 126-137. doi:10.1093/geronb/62.2.p126 
Shire, K. A., Schnell, R., \& Noack, M. (2017). Determinants of outsourcing domestic labour in conservative welfare states: Resources and market dynamics in Germany (No. 2017-04). Duisburger Beiträge zur soziologischen Forschung.

Trukeschitz, B., Schneider, U., Mühlmann, R., \& Ponocny, I. (2012). Informal eldercare and workrelated strain. Journals of Gerontology Series B: Psychological Sciences and Social Sciences, 68(2), 257-267. doi:10.1093/geronb/gbs 101

United Nations, Department of Economic and Social Affairs, Population Division (2019). World Population Prospects 2019, Online Edition.

Van Houtven, C. H., \& Norton, E. C. (2004). Informal care and health care use of older adults. Journal of health economics, 23(6), 1159-1180. doi:10.1016/j.jhealeco.2004.04.008

Wiemers, E. E., \& Bianchi, S. M. (2015). Competing Demands from Aging Parents and Adult Children in Two Cohorts of American Women. Population and Development Review, 4l(1), 127-146. doi:10.1111/j.1728-4457.2015.00029.x 
Table 1. Average indices of positive and negative feelings associated with childcare and adult care obtained by combining data from ATUS and PSID.

\begin{tabular}{cccccc}
\hline & \multicolumn{2}{c}{ Childcare indices } & & \multicolumn{2}{c}{ Adult care indices } \\
\cline { 2 - 3 } \cline { 5 - 6 } Mean & Positive & Negative & & Positive & Negative \\
\cline { 2 - 3 } \cline { 5 - 6 } Ratio & 0.95 & 1.05 & & 4.73 & 1.13 \\
\hline
\end{tabular}


Figure 1. Graphic representation of matrices of time transfers related to caregiving in the US, estimated from the American Time Use Survey (2011-2013).
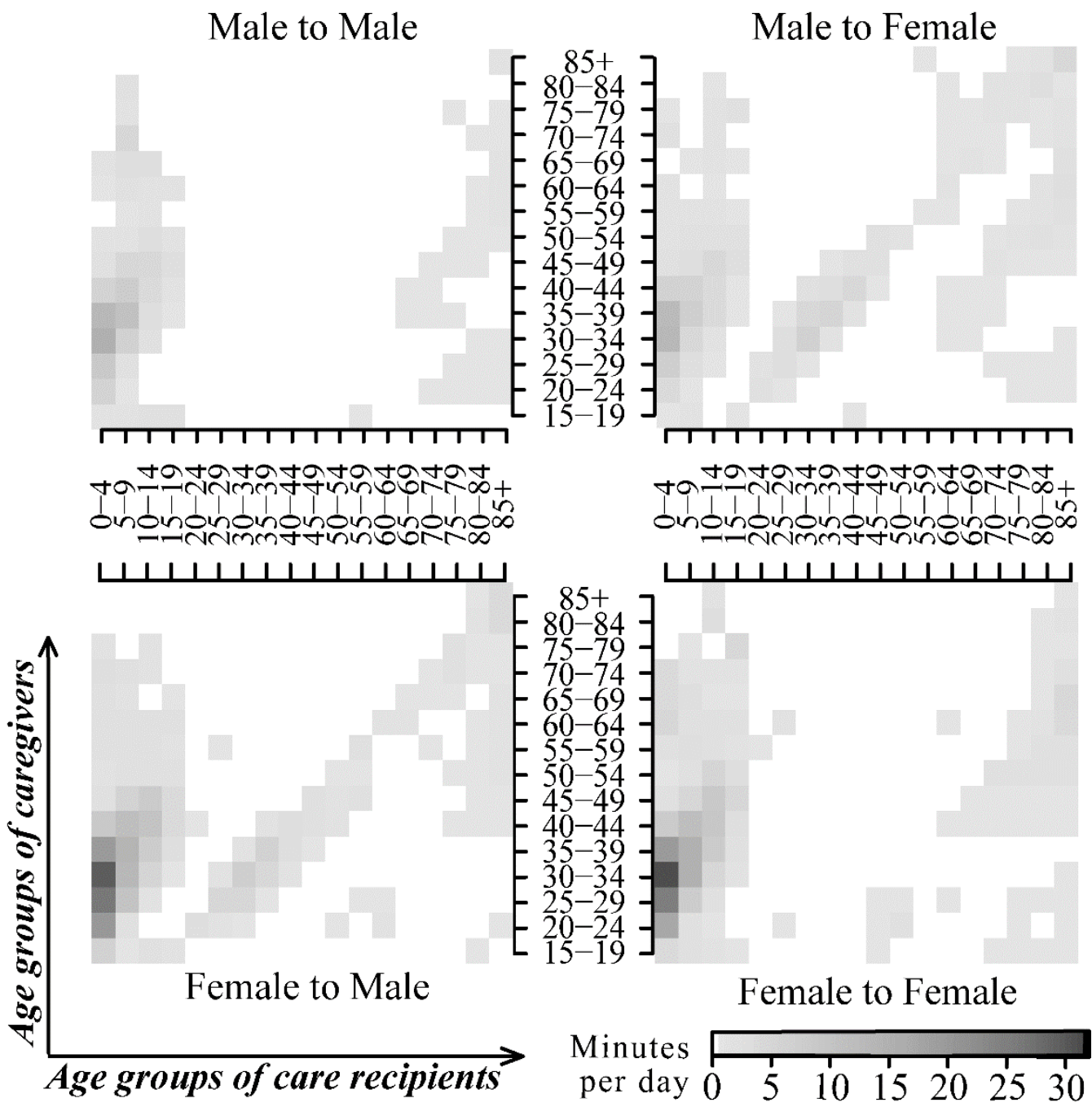

\section{Female to Female}

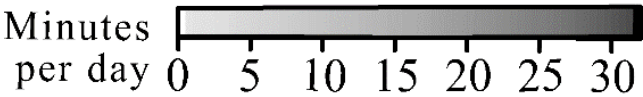


Figure 2. Average ATUS and PSID feeling scores by type of care activity.
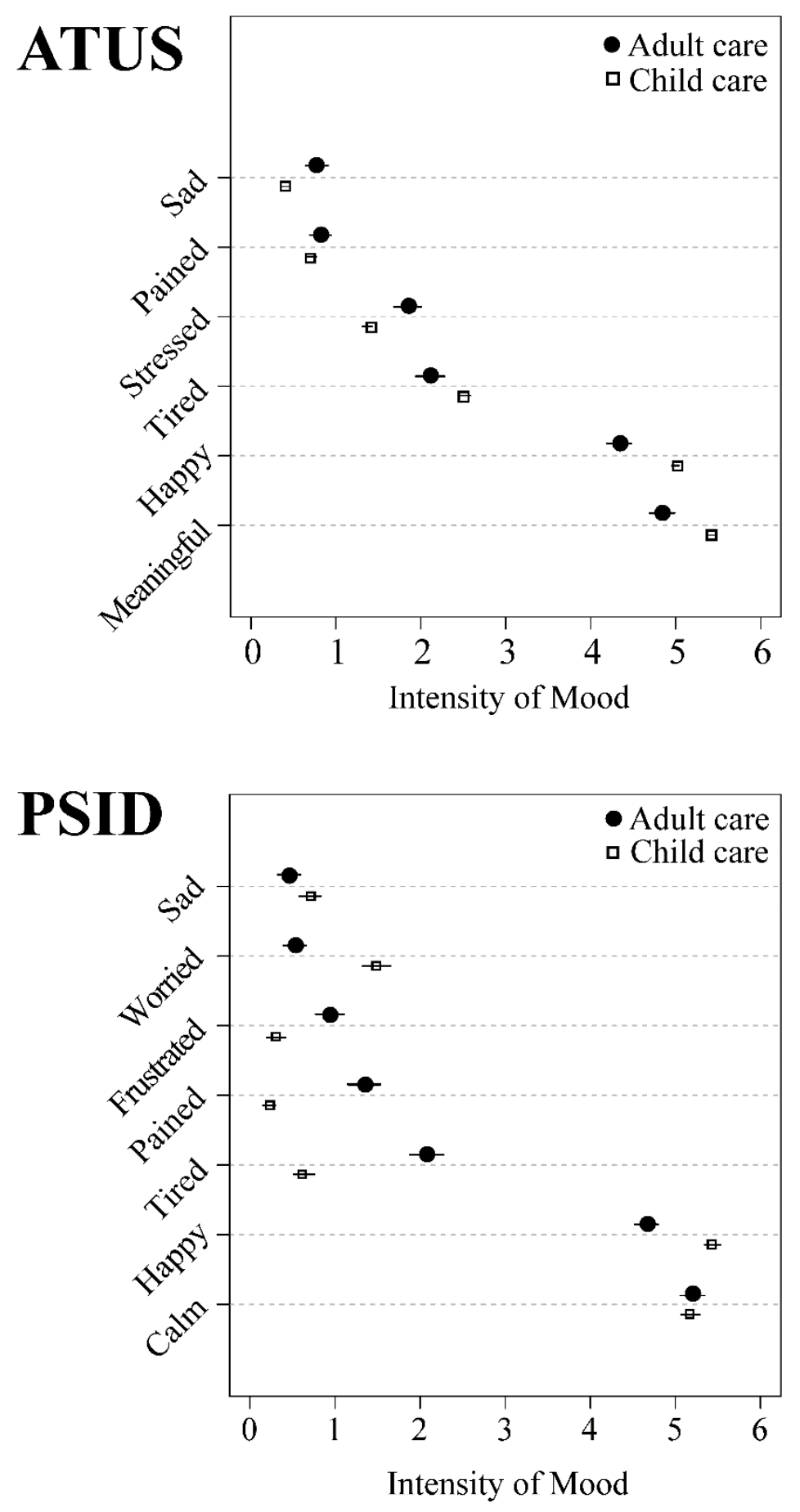

Source: Own elaborations on ATUS/PSID data. Confidence intervals are shown at $95 \%$ level. 
Figure 3. Per capita age profiles of positive and negative care time production.

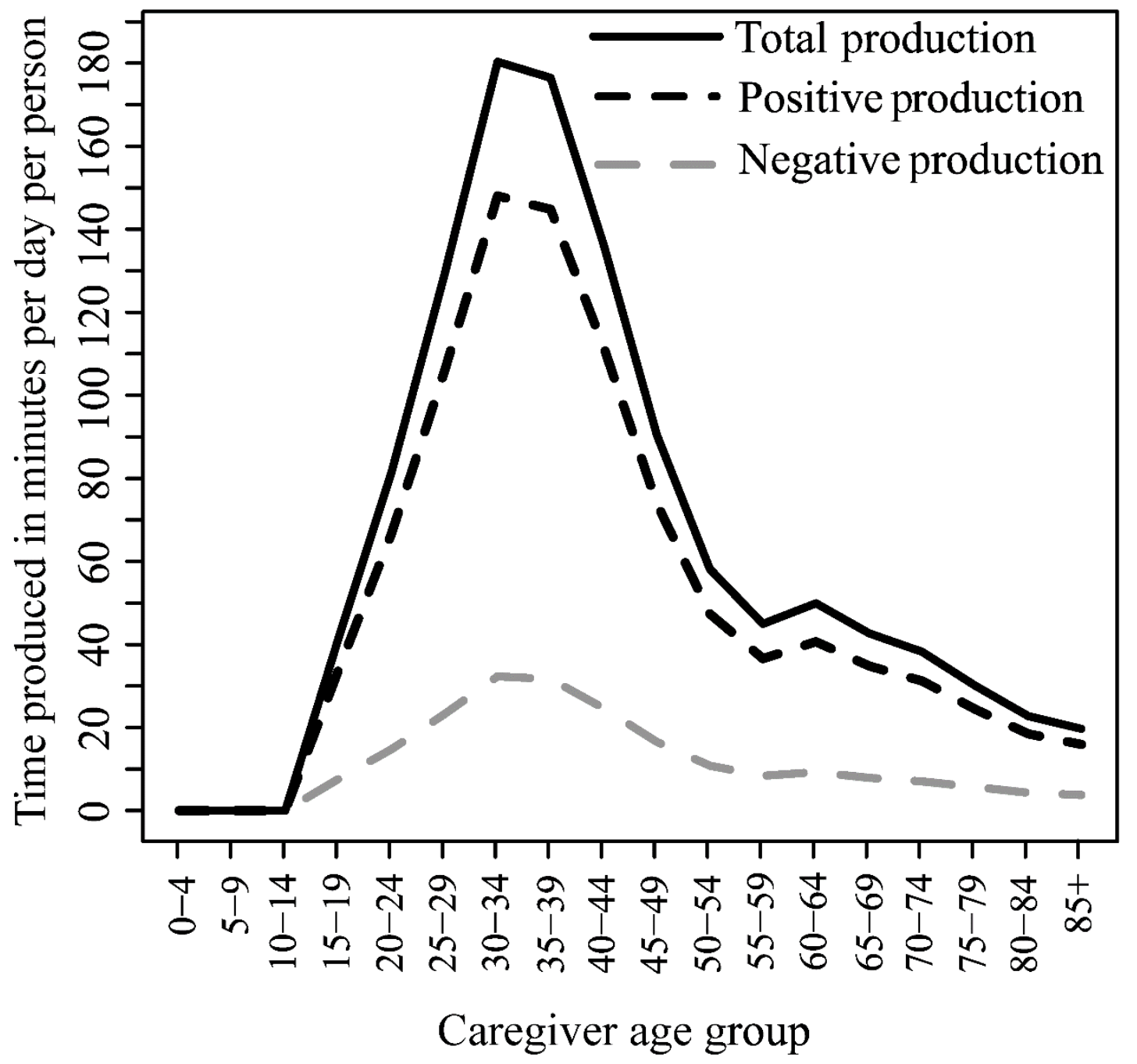


Figure 4. Care support ratios.

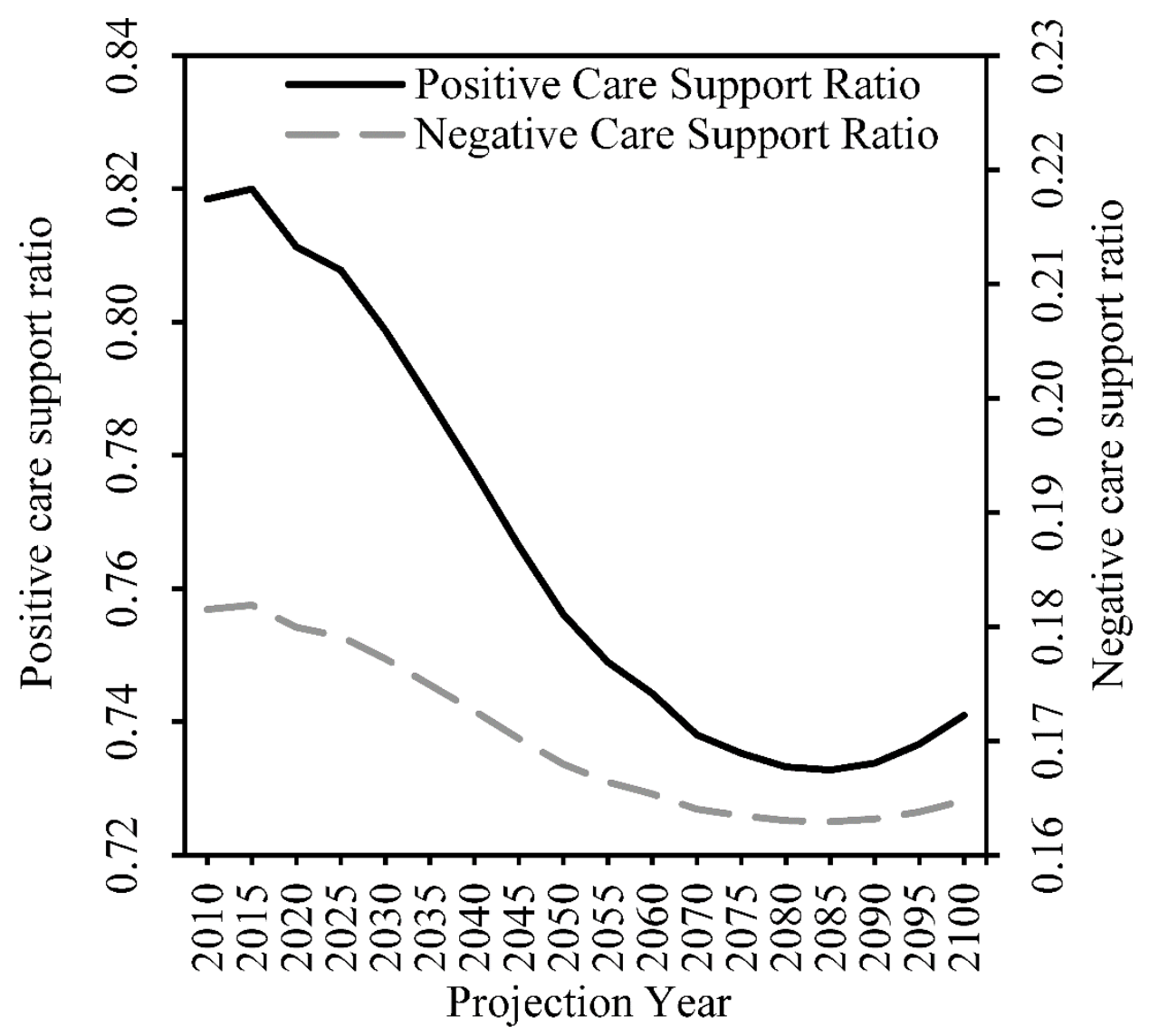

Note: Time is weighted by the positive and negative feelings associated with the care activities.

The sum of the positive and negative care ratios is equal to the total CSR. 


\section{Supplementary Material to "The Impact of Demographic Change on Transfers of Care and Associated Well-being"}

Section 1. Estimating matrices of informal care transfers by age and sex using ATUS data.

To construct the matrices of time transfers, we incorporate information on childcare and adult care transfers using distinct approaches for intra-household and inter-household care time flows. We begin by linking the ATUS Activity File and Who File. Users who download IPUMS ATUS activity-level data extract do not need to perform this step. The Who File contains information about the people present during each activity, which is necessary for us to be able to classify the flow of time spent in care activities by the age and sex of potential care recipients. The Activity File describes the details of each activity, of which duration and classification are the most important for our purposes. If multiple eligible recipients were present (for example, two children in a childcare activity), the time for the activity is split equally among them, as we assume they were equally exposed to care.

Having linked the data and computed the time for each household member, we may now construct the intra-household matrices by age and sex. We chose to aggregate the matrices by standard five-year age groups $(0-4,5-9,10-14$, and so on), as these are the most commonly used groupings for aggregate demographic data. In the matrices, each of which describes care time flows in one of the four transfer combinations by sex (Male-to-Male, Male-to-Female, Female-toMale, Female-to-Female), the cells represent the average daily amount of time spent by caregivers of the corresponding sex and age category (on the rows) caring for care recipients of certain age groups (on the columns). In each sex-to-sex transfer combination represented by a matrix, the averages for every cell are generated according to the ATUS methodology ${ }^{1}$ as follows:

${ }^{1}$ https://www.bls.gov/tus/atususersguide.pdf, p.74 


$$
\bar{T}_{i j}=\frac{\sum_{j} \sum_{i}\left(t_{i} W_{i}\right)_{j}}{\sum_{i} W_{i}}
$$

where $\bar{T}_{i j}$ is the mean amount of time spent caring by a respondent of age group $i$ for care recipients of age group $j$ in an activity that could be childcare, if $j<18$, or adult care, if $j \geq 18$. $t_{i}$ is then the raw number of minutes in each transfer instance, and $W_{i}$ is the survey weight of the caregivers belonging to age group $i$. The activities are broadly classified by the first four digits of the six-digit ATUS activity code, 0301xx, 0302xx, 0303xx, and 0401xx, 0402xx, 0403xx for household and non-household childcare, and 0304xx, 0305xx, and 0404xx, 0405xx for household and nonhousehold adult care; in addition, activity codes 039999 and 049999 are included as miscellaneous help to household and non-household members. Please refer to the ATUS activity lexicon ${ }^{2}$ for detailed descriptions of the activities included in the more general categories used for the matrix construction.

For the inter-household matrices, the approach is less straightforward and needs several modifications. Unfortunately, the main ATUS dataset does not list the age and sex of nonhousehold members, unless they are the respondents' own children. Therefore, we make use of the Eldercare Roster File, which is similar to the Who File used earlier in that we can extract from it the detailed demographic information about non-household members who are eldercare recipients or who receive care due to a condition brought about by aging. Thus, these individuals are typically over age 50 , and more than $90 \%$ of them live outside of the respondents' households. This information is useful, since most inter-household adult care transfers occur in this age category. As for children under 18 who are not the caregivers' own children and are living outside of their households, we assume that the average amount of care time these children received was similar

${ }^{2}$ https://www.bls.gov/tus/lexiconwex2013.pdf 
to that received by their own non-household children. It is a weak assumption that does not entail deviations or bias of a large magnitude, as inter-household childcare provided to non-own children is fairly rare. Finally, for non-elderly adults, such as non-co-resident neighbors or friends, we split the time equally across all age/sex groups of inter-household care recipients aged 18-49. Additionally, to refine the estimates for middle age inter-household adult care, we assume that any member who is identified as a non-co-resident spouse belongs to the same age group as the caregiver, but is of the opposite sex.

Assembling all the components together and adding the numerators of the intra- and interhousehold matrices before dividing by the corresponding caregiver weights (see equation above) results in the overall informal care time transfers matrices shown in Figure 1 of the manuscript.

Section 2. Formal theoretical framework for generating per capita age schedules of positive and negative care time production.

In the context of a stable equilibrium, the profiles of care time production by age can be obtained or interpreted as the leading eigenvector of the matrix of fractions of time production for the receiving group. Here, we explain the intuition that informs this approach.

Consider a population with $m$ age groups, indexed by $i$. Total time production (positive or negative) for age group $i, t_{i}$, is written as:

$$
t_{i}=z_{i} 1+z_{i} 2+\cdots+z_{i m}
$$

where $z_{i j}$ is the time flow from group $i$ to group $j$. The expression can be rewritten to represent the flows between groups as the fraction of total time production for the receiving group. Thus, if we let $q_{i j}=\left(z_{i j} / t_{j}\right)$, the model is expressed as:

$$
t_{i}=q_{i 1} t_{1}+q_{i 2} t_{2}+\cdots+q_{i m} t_{m}
$$

or, equivalently, as: 


$$
-q_{i 1} t_{1}-q_{i 2} t_{2}-\cdots+\left(1-q_{i i)}\right) t_{i}-\cdots-q_{i m} t_{m}=0
$$

By letting $Q$ be the $m \times m$ matrix containing all the coefficients $q_{i j}$, and $T$ be the $m \times 1$ vector containing all the transfer $t_{i}$ terms, the model is written in a more compact form as:

$$
[I-Q] T=0
$$

or, equivalently, as:

$$
Q T=T
$$

The solution of Equation 5 states that $T$ is the eigenvector of $Q$ associated with the leading eigenvalue. In other words, per capita profiles of production by age in steady state can be interpreted as the eigenvector of a matrix of the type of $Q$ associated with the leading eigenvalue. This result is general and applies to various classes of production profiles, including the standard ones generated within the context of the National Transfer Accounts (NTA) project. Figure 4 presented in the main manuscript is the result of this approach.

Section 3. The impact of demographic change on quality-adjusted care time.

Using the quality-adjusted indices of dependency computed earlier, we evaluated the potential implications of the expected changes in the composition of the US population, according to the United Nations projections, on the aggregate care. We began with the matrices presented in Figure 1. We first multiplied all care time consumed by the $0-4,5-9$, and 10-14 age groups by the positivity/negativity ratios generated for childcare; and all other age groups by the ratios for adult care. Although individuals in the 15-19 age group were technically considered children, we treated them as adults for this specific procedure, since children in their late teens typically do not require the same level of attention from their parents as their younger counterparts. As a result, the overall matrix of time transfers was split into two components: a matrix depicting flows of positive time 
and another representing flows of negative care time. The two matrices were designed to sum up to the original matrix of overall time transfers.

The matrices disaggregated by positive and negative states highlight the large differences in time production by sex. Women, who dedicated more time to caregiving than men, had higher exposures to both positive and negative care time. Overall, the magnitude of positive time was larger than the magnitude of negative time for both men and women. Parents who were caring for their children reported the most positive experiences while the children were under age four, but this positivity tapered off as the children grew older. Even elderly individuals, probably grandparents, experienced childcare as generally positive, whereas most respondents reported that caring for teenagers between ages 15-19 was a negative experience. In addition, a large share of the caregiving perceived as negative was between caregivers and care recipients of similar ages, which suggests that they were spouses. It is possible that this negativity was due to circumstances in which one spouse required the care of the other, such as in the aftermath of an accident or a health issue. Situations in which a middle-aged adult was caring for an elderly parent (aged 75 or older), or in which an elderly individual was caring for an elderly spouse, were also generally seen as negative experiences. 